\title{
Scaling of dot dispersion from directional rank order data
}

\author{
HIROSHI HOJO ${ }^{1}$ \\ Department of Educational Psychology, School of Education, Waseda University, Shinjuku-ku, Tokyo 160
}

\begin{abstract}
Subjective impression of dispersion of 60 or 300 dots scattered in an area of $8.2 \times 8.2(\mathrm{~cm})$ was scaled by a nonmetric maximum likelihood scaling method for directional rank order data. The rank order data were analyzed under all combinations of three types of representation models of subjective impression of dot dispersion and five kinds of physical variables of stimuli. The analysis has revealed that the quasi-psychophysical function model (QFM) based on the mean weighted area of a triangle determined by any two dots and the centroid of all dots is the most appropriate representation model. QFM assumes that a major part of the variance in the data can be accounted for by Fechner's law, but that the remaining portion whose relationships with physical variables have not been made explicit should be represented by correction parameters (i.e., a small set of real numbers to be assigned to each stimulus).
\end{abstract}

Key words: psychophysical scaling, maximum likelihood estimation, directional rank orders, dot dispersion, Akaike's information criterion (AIC).

A look at such dot patterns as shown in Fig. 1 gives us some impression of dispersion of dots scattered in a two dimensional space. We can also detect the differences between patterns in the extent to which their dots are spread out. Thus, perceiving dispersion of objects may be as general as perceiving numerousness of objects, or as perceiving brightness, loudness, etc. This suggests that it is possible to scale the subjectively experienced dispersion of dots.

An attempt to scale the impression of dot dispersion, however, will give rise to an interesting problem which would not arise in the case of scaling dot numerosity (Indow \& Ida, 1977). That is the problem of identifying the physical variable eliciting the subjective impression of dot dispersion. In perception of dot numerosity the objective number of dots is almost uniquely under effect for all subjects, all other things being equal, whereas it is open to question what physical dimensions are really relevant when we judge dot dispersion. The purpose of the present

1 The author wishes to express his gratitude to Professor Tatsuro Makino of Waseda University for his useful advice during preparation of this paper. study is not only to scale the subjective impression of dot dispersion but also to find what physical variables are utilized in judging the degree to which dots are spread out.

Our general approach is in line with recent developments in multidimensional scaling (e.g., Takane, 1981; Takane \& Carroll, 1981; Takane \& Sergent, 1983). According to their method a set of stimuli are assumed to have some parametric representation. In the present context the representation model assigns a scale value to each stimulus. The scale value is then assumed to be error-perturbed in a specific way. The nature of this perturbation process is captured in the error model. The error-perturbed scale value is then related to a specific task situation through a model of psychological processes. The specific task situation generates a specific form of data, and the response model specifies the nature of relationship between the observed data and the underlying processes. Thus, once all the relevant component models are specified, the likelihood of observed data can be stated in terms of parameters in these models. Maximum likelihood estimation can then be used to determine the estimates of the 
parameters (Takane \& Sergent, 1983). In passing, however, we may point out that the present method has a somewhat different feature from theirs. That is, this study examines the validity of adopting a psychophysical law (Fechner's law) as the representation model of dot dispersion by comparing it with other models which assume no specific psychophysical relationship such as Fechner's law.

\section{The Models}

\section{The Representation Model}

As candidates for the representation model of the subjectively experienced dot dispersion we choose three different types of models: the psychophysical function model, the unconstrained model, and the quasi-psychophysical function model.

The psychophysical function model (PFM). A psychophysical function assumed here is Fechner's logarithmic function (Luce \& Galanter, 1963), which is of the form

$$
\begin{aligned}
d_{i} & =A \ln \left(s_{i k}+\gamma\right)+B \\
(i & \left.=1, \ldots, n ; k=1, \ldots, \mathcal{N}_{k}\right),
\end{aligned}
$$

where $d_{i}$ is the scale value of stimulus $i$ representing the impression of dot dispersion, $A$ is a unit parameter of the scale, $s_{i k}$ is the value of stimulus $i$ on physical variable $k, \gamma$ and $B$ are constants, $n$ is the number of stimuli, and $\mathcal{N}_{k}$ is the number of physical variables to be considered.

The unconstrained model (UCM). The unconstrained model assumes neither specific psychophysical relationships nor further structures than each stimulus has its own scale value independent of any physical measure of stimuli. Therefore, UCM is expressed as a vector of $n$ scale values each assigned to the corresponding $n$ stimuli like

$$
\boldsymbol{d}=\left(d_{1}, \ldots, d_{n}\right) .
$$

Note that the model is free to assume $d_{i}=d_{j}$ for any stimuli $i$ and $j$ if they are close enough so as to reduce the number of distinct scale values for dispersion. The number of distinct values will be designated by $\mathcal{N}_{d}$.

The quasi-psychophysical function model $(Q F M)$. We propose another type of UCM called the quasi-psychophysical function model. In this model dispersion parameter $d_{i}$ given in UCM is restricted in such a way that

$$
d_{i}=f\left(s_{i k}\right)+c_{i k},
$$

where $f\left(s_{i k}\right)$ is the psychophysical function defined by (1), and $c_{i k}$ is a correction parameter which represents the fraction of dispersion value $d_{i}$ that cannot be accounted for by the corresponding PFM. Indices $i$ and $k$ in $c_{i k}$ indicate stimulus $i$ and physical variable $k$, respectively. Although $c_{i k}$ is specific to each stimulus $i$, we may again impose a restriction $c_{i k}=c_{j k}$ for any $i$ and $j$ if they are close enough. This reduces the number of correction parameters. Let us call a group of stimuli sharing a common value of correction parameters the correction class, and denote the parameter value of correction class $l$ by writing $C_{l}$. Then, $c_{i k}=C_{l}$ if stimulus $i$ belongs to correction class $l$. The effective number of correction parameters equals to the number of correction classes, $\mathcal{N}_{c}$, minus 1 since, say, $C_{1}$ can be set to zero without loss of generality. We expect that in ordinary circumstances the number of correction parameters needed in QFM tends to decrease as the proportion of scale value $d_{i}$ accounted for by PFM increases, because the variability in values of $C_{l}$ would then decrease as the amount of disparities between true values of correction parameters becomes smaller.

It is obvious that QFM is reduced to PFM when $\mathcal{N}_{c}=1$. On the other hand, QFM is equivalent to the simple UCM when $\mathcal{N}_{c}=\mathcal{N}_{d}$. In this sense PFM and UCM can be considered as special cases of QFM.

In what follows we will employ abbreviations $\mathrm{PFM}_{k}$ and $\mathrm{QFM}_{k}$ to designate PFM and QFM, respectively, with physi- 
cal variable $k$.

Now, let us discuss the question of what physical variables of stimuli we should take into consideration for PFM and QFM. In this analysis the following five kinds of variables will be considered:

1. The mean weighted area of a triangle determined by any two dots and the centroid of a set of all dots plotted.

2. The mean weighted distance from the centroid to each dot.

3. The mean log distance from the centroid to each dot.

4. The mean distance among all dots.

5. The area of the smallest polygon that contains all dots in it.

Let index $k(k=1,2,3,4$, and 5) refer to the five physical variables. Then, $s_{i k}$, the value of stimulus $i$ on each variable is formally defined as follows:

$$
\begin{aligned}
& s_{i 1}=\frac{1}{\mathcal{N}(\mathcal{N}-1) / 2} \sum_{g<h} W_{g h l} A_{g h} \\
& (g=1, \ldots, \mathcal{N}-1 ; h=2, \ldots, \mathcal{N}),
\end{aligned}
$$

where

$$
W_{g h}=A^{b}{ }_{g h},
$$

and where $\mathcal{N}$ is the number of dots, $A_{g h}$ is the area of a triangle determined by any two dots $g, h$ and the centroid of all dots, and $b(\geqq 0)$ is a constant. Evidently in this definition the weight $W_{g h}$ is assumed to be a power function of $A_{g h}$ itself.

$$
s_{i 2}=\frac{1}{\mathcal{N}} \sum_{j=1}^{N} W_{j} D_{j},
$$

where

$$
W_{j}=D^{u}{ }_{j},
$$

and where $D_{j}$ is the distance from the centroid to $\operatorname{dot} j$, and the weight $W_{j}$ is a power function of $D_{j}$ itself in this formula, too.

$$
s_{i 3}=\frac{1}{N} \sum_{j=1}^{N} \ln D_{j} .
$$

$$
\begin{gathered}
s_{i 4}=\frac{1}{\mathcal{N}} \sum_{j=1}^{N} \frac{1}{\mathcal{N}-1} \sum_{h \neq j} G_{j h} \\
(j=1, \ldots, \mathcal{N} ; h=1, \ldots, \mathcal{N}),
\end{gathered}
$$

where $G_{j h}$ is the distance between $\operatorname{dots} j$ and $h$.

$$
s_{i 5}=a_{i},
$$

where $a_{i}$ represents the area of the smallest polygon that contains all dots making up stimulus $i$.

\section{The Error Model}

The following error model, which is called the additive error model (Takane, 1981), will be considered in this analysis:

$$
\left\{\begin{array}{l}
\lambda^{(t)}{ }_{i r}=d_{i r}+e^{(t)}{ }_{i r} \\
e^{(t)}{ }_{i r} \sim \mathcal{N}\left(0, \sigma^{2}\right),
\end{array}\right.
$$

where $\lambda^{(t)}{ }_{i r}$ is an error-perturbed metric process for replication $r$ and at occasion $t$.

\section{The Response Model}

We collected the data by the directional ranking method. In this method ranking is performed in a specific direction, to take the present example, from a stimulus pattern with the most closely clustered dots around the centroid of all dots to the one with dots which are most widely spread out. The method is directional in this sense. Ordinarily the subject is presented with $n$ stimuli at a time. In the present context he (or she) is first instructed to choose the dot pattern whose dots are most closely gathered around the center of the pattern among all $n$ stimuli. Then after this pattern is eliminated he is required to choose the stimulus which has now the most closely clustered dots among the $n-1$ remaining patterns, and so on until a complete ranking is obtained among the $n$ stimuli.

Takane and Carroll (1981) have developed a maximum likelihood multidimensional scaling method for such directional rank order data. Our response model can be viewed as a special case of 
theirs in that ours is for a unidimensional scaling. The following is only a brief description of the model. For a more comprehensive description, see their original paper.

Let $d_{m r}$ denote the model prediction of the stimulus which is judged to have the $m^{\text {th }}$ most closely gathered dots in replication $r$. Let $\lambda^{(t)}{ }_{m r}$ represent the errorperturbed model prediction corresponding to $d_{m r}$ generated at occasion $t$. The occasion in this case refers to each successive first choice. Then, the probability, $p^{(m)} r$, that the stimulus corresponding to $d_{m r}$ is chosen as the one with the $m^{\text {th }}$ most closely clustered dots is given by

$$
\begin{aligned}
& p^{(m)}{ }_{r}=\operatorname{Pr}\left(\lambda^{(m)}{ }_{m r}<\lambda^{(m)}{ }_{m+1, r}, \ldots,\right. \\
& \left.\lambda^{(m)_{m r}}<\lambda^{(m)}{ }_{n r}\right) \text {, }
\end{aligned}
$$

which can be well approximated by the multivariate logistic distribution

$$
p_{r}^{(m)}=\left[1+\sum_{j=m+1}^{n} \exp \left\{v\left(d_{m r}-d_{j r}\right)\right\}\right]^{-1},
$$

where $v$ is a dispersion parameter which is approximately $\pi /(\sqrt{6} \sigma)$ (Takane \& Carroll, 1981).

The probability of a complete ranking of $n$ stimuli in replication $r, p_{r}$, is given by

$$
p_{r}=\prod_{m=1}^{n} p^{(m)}
$$

where for $m=n$ we have $p_{r}^{(n)}=1$. The joint likelihood for the entire set of observations is stated as

$$
L=\prod_{r=1}^{R} p_{r},
$$

where $R$ is the number of replications. The $\log$ of $L$ is maximized with respect to parameters in the models (e.g., $A, \gamma, b, C_{l}$ $\left(l=1, \ldots, \mathcal{N}_{c}\right)$, and $\sigma$ for $\left.\mathrm{QFM}_{1}\right)$ by a quasi-Newton method in this analysis. A BASIG program of this numerical method is taken from Tone (1981).

In evaluating the goodness of fit of the models we use the AIC statistic (Akaike,
1974) defined by

$$
\operatorname{AIC}(\phi)=-2 \ln L+2 n_{\psi},
$$

where $n_{\psi}$ is the effective number of parameters in model $\psi$. Only relative magnitudes of AIG are meaningful. (The model associated with a smaller value of AIC is considered the better model.) If we use notations $n_{P}, n_{U}$, and $n_{Q}$ to denote the effective numbers of parameters necessary for models PFM, UCM, and QFM, respectively, then they are each evaluated by

$n_{P}=1($ for $A)+1($ for $\gamma)+1($ for $\sigma)-1$ (for arbitrary unit of the scale value) + 1 for $b$ (only when $k=1$ or $k=2$ ) \} (Note that constant $B$ in model (1) does not appear in the response model given above. See equation (11).),

$n_{b l}=\mathcal{N}_{l}+1$ (for $\left.\sigma\right)-1$ (for arbitrary unit of the scale value), and

$$
n_{Q}=n_{P}+\mathcal{N}_{c}-1 \text {. }
$$

The estimate by the above procedure is generally called the minimum information theoretic criterion (AIC) estimate (MAICE) (Akaike, 1974). The major advantage of this method is that it enables us to empirically choose the most appropriate model from among several competitive ones by comparing their goodness of fit. For example, we are able to compare the goodness of fit of PFM with that of UCM, which would not have been impossible in classical psychophysics.

\section{An Example of Application}

\section{The Data}

A small experiment was carried out to collect relevant data. Stimuli were dot patterns as shown in Fig. 1. Dot patterns were of two types: One was the type which was made up by a set of 60 dots. The other was the one made up by a set of 300 dots. They will be called 60- and 300-dot type, respectively. These stimuli 
were generated by a microcomputer. First, $\mathcal{N}$ dots were randomly plotted in a 70 by 70 unit matrix (a $8.2 \times 8.2(\mathrm{~cm})$ plane) with a certain degree of dispersion,

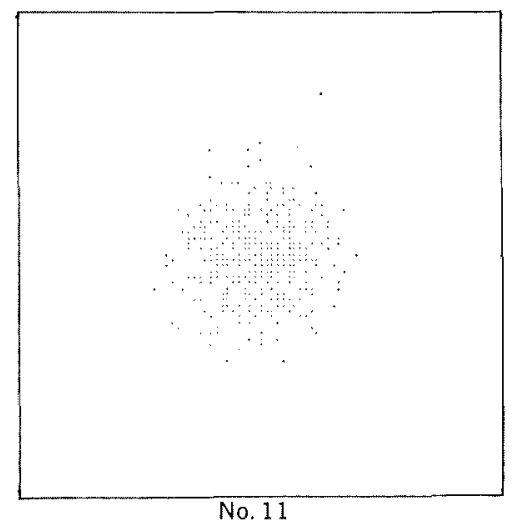

(a)

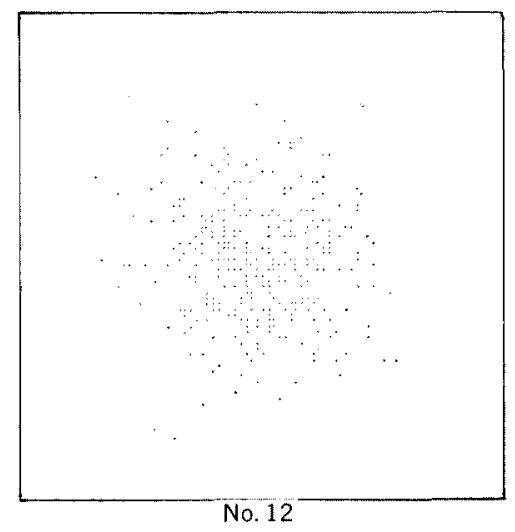

(b)

Fig. 1. Samples of stimulus dot patterns No. 11 in (a) and No. 12 in (b) for 300-dot type. Their (raw) values on the five physical variables $\left(s_{i k}\right)$ are as follows:

\begin{tabular}{lcc}
\hline $\begin{array}{c}\text { Physical } \\
\text { variable } \\
k\end{array}$ & (a) & Patterns \\
\hline 1 & $62156^{\text {a) }}$ & 544407 \\
2 & $18461^{\text {b) }}$ & 78323 \\
3 & 2.244 & 2.593 \\
4 & 15.29 & 21.98 \\
5 & 1388 & 3182 \\
a) The value calculated when $\mathrm{b}=1.89$ (see text \\
and Table 1). \\
b) The value calculated when $\mathrm{b}=2.76$.
\end{tabular}

which was copied on a $12 \times 12(\mathrm{~cm})$ card with a $10.2 \times 10.2(\mathrm{~cm})$ square frame. This card served as a stimulus (see Fig. 1). For each dot type 16 such stimuli were sampled. They were quasi-randomly distributed along each physical variable under comparison.

Thirty-three and 32 college students participated in the experiment for 60- and 300-dot types, respectively. A set of 16 stimuli $(n=16)$ were presented at a time in a row on the table. Subjects were instructed to rank these 16 dot patterns according to the degree of clustering of their dots by the directional ranking method.

\section{Identifying the Best Fitting Model}

The three representation models to be compared were fitted to the rank order data. There are two points to be noted regarding applications of the models: (a) A set of $16 s_{i k}(i=1, \ldots, 16)$ values were standardized in such a way that they had a mean of 5 and a standard deviation of 1 for each $k$. For simplicity of notation, however, symbol $s_{i k}$ will be used to represent the standardized values as well as raw values. (b) Subjects were taken as replications of a single subject assuming that there are no substantial individual differences in perception of dot dispersion $(R=33$ or $R=32$ ).

We first fitted UCM to the data. The results are reported in Table 1. The values of $\mathrm{AIC}$ obtained for UCM under several assumptions about the number of distinct scale values $\left(\mathcal{N}_{d}\right)$ indicate that for 60-dot type the $\mathcal{N}_{d}=10$ model assuming $d_{1}=d_{15}, \quad d_{3}=d_{13}, \quad d_{4}=d_{16}, \quad d_{6}=d_{7}=d_{8}, \quad$ and $d_{10}=d_{12}$, and for 300-dot type the $\mathcal{N}_{d}=13$ model assuming $d_{2}=d_{15}, d_{5}=d_{13}, d_{8}=d_{9}$ are the best fitting unconstrained models.

The summary of analysis by PFM and QFM is also presented in Table 1 , and further depicted in Fig. 2, and Fig. 3. However, the AIG values of $\mathrm{PFM}_{k}$ and $\mathrm{QFM}_{k}$ for $k=3,4$, and 5 are omitted from the table. It is because from the results 
Table 1

Summary of applications of MAICE to the rank order data of 16 dot patterns with varying dot dispersions

\begin{tabular}{|c|c|c|c|c|c|c|}
\hline $\begin{array}{l}\text { Representation } \\
\text { models }\end{array}$ & $\begin{array}{l}\text { Equality assumptions on } \\
\text { dispersion or } \\
\text { correction values }\end{array}$ & $\gamma$ & $b$ & $\sigma^{\mathfrak{a} \gamma}$ & $n_{\ddot{\phi}}$ & $\mathrm{AIC}$ \\
\hline \multicolumn{7}{|l|}{$60-d o t$ type } \\
\hline $\operatorname{UGM} \mathcal{N}_{t l}=9$ & $\begin{array}{l}d_{1}=d_{15}, \quad d_{3}=d_{10}=d_{12}=d_{13}, \\
d_{4}=d_{16}, \quad d_{6}=d_{7}=d_{3},\end{array}$ & & & .34 & 9 & 1141.3 \\
\hline $\mathcal{N}_{d}=10$ & $\begin{array}{l}d_{1}=d_{15}, \quad d_{3}=d_{13}, \quad d_{4}=d_{16} \\
d_{6}=d_{7}=d_{8}, \quad d_{10}=d_{12}\end{array}$ & & & .34 & 10 & 1132.7 \\
\hline $\mathcal{N}_{d}=11$ & $\begin{array}{l}d_{1}=d_{15}, d_{3}=d_{13}, d_{1}=d_{16} \\
d_{6}=d_{7}, d_{10}=d_{12}\end{array}$ & & & .34 & 11 & 1133.3 \\
\hline $\mathrm{PFM}_{1}$ & & -3.58 & 1.86 & .41 & 3 & 1150.9 \\
\hline $\mathrm{PFM}_{2}$ & & -2.69 & 1.89 & .21 & 3 & 1161.1 \\
\hline $\mathrm{QFM}_{1} \mathcal{N}_{c}=4$ & $\begin{array}{l}C_{1}=c_{4}^{\mathrm{b})}=c_{8}=c_{12}, C_{2}=c_{2} \\
=c_{3}=c_{10}=c_{11}=c_{15}, C_{3}=c_{6} \\
=c_{9}=c_{13}=c_{14}=c_{10} \\
C_{4}=c_{1}=c_{5}=c_{7}\end{array}$ & -3.57 & 1.86 & .39 & 6 & 1123.7 \\
\hline $\mathrm{QHM}_{2} \mathcal{N}_{c}=4$ & $\begin{array}{l}C_{1}=c_{6}=c_{10}=c_{11}=c_{12}=c_{16} \\
C_{2}=c_{2}=c_{3}=c_{1}=c_{8}=c_{15} \\
C_{3}=c_{5}=c_{7}=c_{14} \\
C_{4}=c_{1}=c_{9}=c_{13}\end{array}$ & -2.26 & 1.92 & .17 & 6 & 1124.6 \\
\hline \multicolumn{7}{|l|}{$300-d o t$ type } \\
\hline $\operatorname{UCM} \mathcal{N}_{a}=12$ & $d_{2}=d_{15}, d_{5}=d_{6}=d_{13}, d_{9}=d_{9}$ & & & .29 & 12 & 914.7 \\
\hline $\mathcal{N}_{t}=13$ & $d_{2}=d_{15}, d_{5}=d_{13}, d_{8}=d_{9}$ & & & .29 & 13 & 913.7 \\
\hline $\mathcal{N}_{L}=14$ & $d_{2}=d_{15}, d_{5}=d_{13}$ & & & .29 & 14 & 915.0 \\
\hline $\mathrm{PFM}_{1}$ & & -3.59 & 1.89 & .29 & 3 & 928.5 \\
\hline $\mathrm{PFM}_{2}$ & & -2.92 & 2.45 & .17 & 3 & 926.9 \\
\hline $\mathrm{QFM}_{1} \mathcal{N}_{l}=4$ & $\begin{array}{l}C_{1}=c_{3}=c_{7}=c_{9}=c_{12}=c_{13}=c_{11} \\
=c_{16}, C_{2}=c_{1}=c_{2}=c_{6}=c_{10}=c_{11} \\
=c_{15}, C_{3}=c_{4}=c_{5}, C_{4}=c_{8}\end{array}$ & -3.59 & 1.89 & .28 & 6 & 901.4 \\
\hline $\mathrm{QHM}_{\mathrm{y}} \mathcal{N}_{c}=4$ & $\begin{array}{l}C_{1}=c_{2}=c_{3}=c_{7}=c_{0}=c_{12}=c_{13}= \\
c_{16}, C_{2}=c_{1}=c_{6}=c_{10}=c_{11}=c_{15}, \\
C_{3}=c_{4}=c_{5}=c_{14}, C_{4}=c_{8}\end{array}$ & -2.86 & 2.76 & .16 & 6 & 901.7 \\
\hline
\end{tabular}

a) For PFM and QFM the estimates obtained by setting $A=1$ are given.

b) Physical variable index $k$ is omitted in $c_{i k}$ here.

of rough evaluations of their values (for example, $\mathrm{AIC}\left(\mathrm{PFM}_{4}\right)=935.4$, and $\mathrm{AIC}$ $\left(\mathrm{QFM}_{4}\right)=902.8$ for 300-dot type) we judged these variables as irrelevant. The degree of fits may be detected to a certain extent in Fig. 2, where scale values estimated from the $\mathcal{N}_{d}=n$ UGM are plotted against these physical variables. From the table it is seen that for both dot types $\mathrm{QFM}_{1}$ consistently shows the minimum AIC values, indicating the best fit, although the differences in AIC between $\mathrm{QFM}_{1}$ and $\mathrm{QFM} \mathrm{M}_{2}$ are small. (The former has the AIC value of 901.4 and the latter 901.7 for 300-dot type, for example.) Figure 3 shows the plots of scale values predicted from these minimum AIC solutions as a function of physical variable 1 . The four correction parameter values used in $\mathrm{QFM}_{1}$ are $.000, .074,-.097$, and -.159, for $\mathrm{C}_{1}, \mathrm{C}_{2}, \mathrm{C}_{3}$, and $\mathrm{C}_{4}$, respectively, in the case of 60-dot type, and they are .000 , $.104,-.085$, and -.229 for 300 dot-type. The additive constant $B$ in $\mathrm{QFM}_{1}$, on the other hand, was determined so that the smallest scale value might be roughly 
equal to that estimated from the $\mathcal{N}_{d}=n$ UCM. They are 2.164 and 2.185 for 60- and 300-dot types, respectively. See Table 1 for the remaining parameter values.

To summarize, the following three observations can be made from the present analysis :
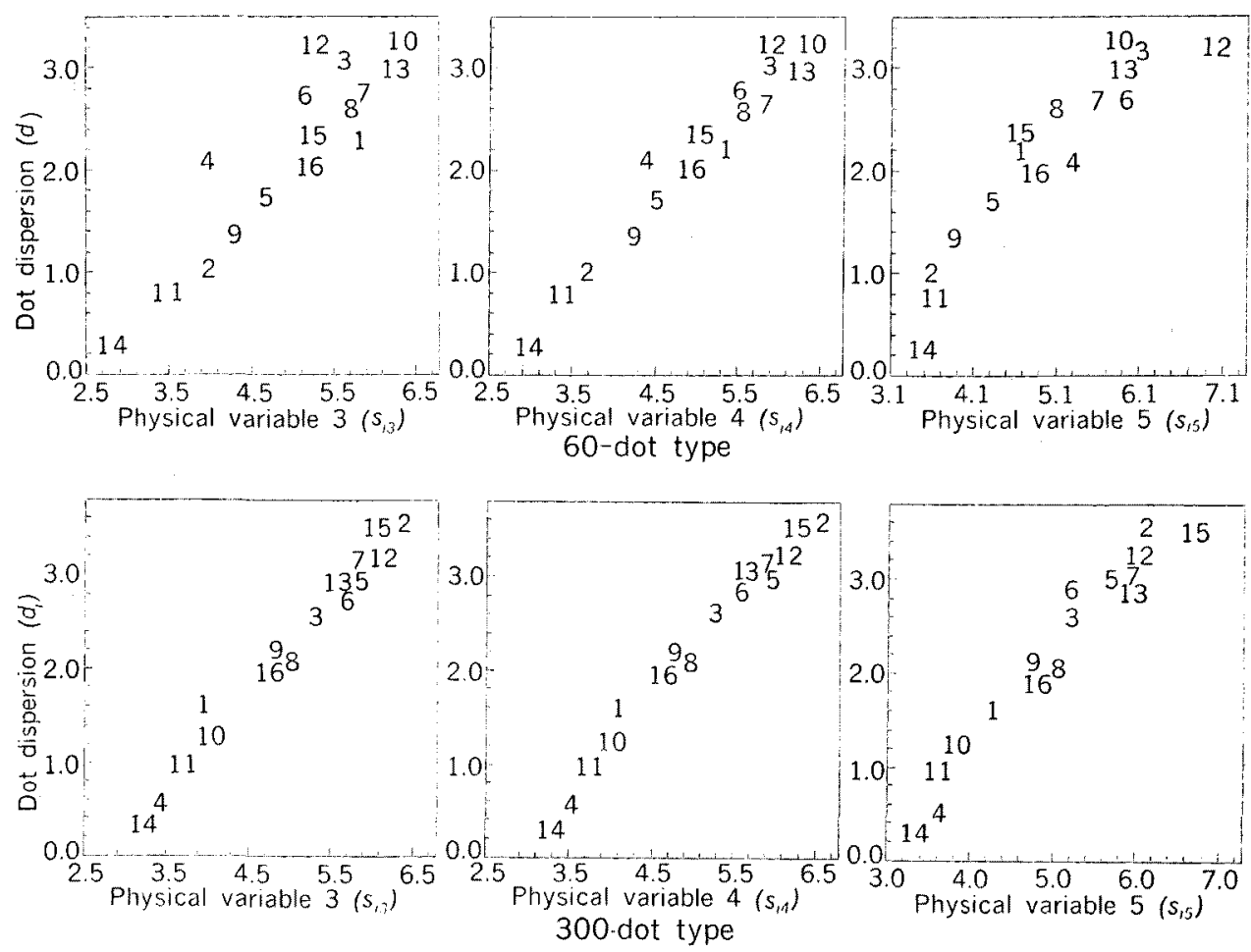

Fig. 2. Scale values of 16 stimuli (stimulus numbers are $1, \ldots, 16$, respectively) estimated from the $\mathcal{N}_{d}=16$ unconstrained model are plotted as a function of each physical variable 3, 4, and 5 for 60 -dot type (upper panels) and 300-dot type (lower pancls).

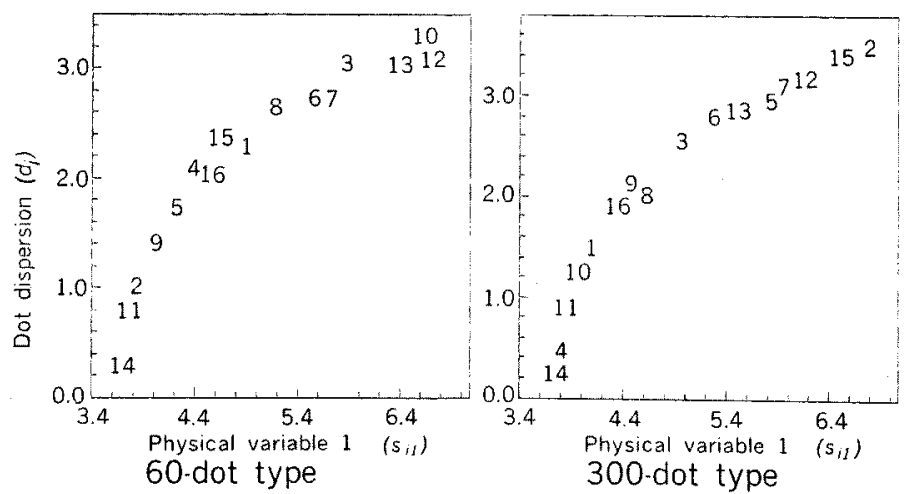

Fig. 3. Scale values of 16 stimuli (stimulus numbers are $1, \ldots, 16$, respectively) estimated from $Q^{2} F M_{1}$ are plotted as a function of physical variable 1 for 60-dot type (left panel) and 300-dot type (right panel). 
(a) The differences in AIC between $\mathrm{QFM}_{1}$ and $\mathrm{QFM}_{2}$ are so small that it seems difficult to definitely conclude which is the more appropriate for the representation model (see "discussion"). Such a result may have come from the fact that two physical variables 1 and 2 are closely related to each other; the Pearson productmoment coefficients of correlation between $s_{i 1}$ and $s_{i 2}$ were .986 and .993 for $60-$ and 300 -dot types, respectively, which suggests that the correlation coefficient approaches to one as the number of dots increases, and that variable 1 will be identical with variable 2 when $\mathcal{N}=\infty$. At the moment, however, it is rational to consider physical variable 1 as the more relevant in perception of dot dispersion at least when $\mathcal{N}$ varies from 60 to 300 , mainly on the ground that in the case of 60-dot type $\mathrm{PFM}_{1}$ gave the distinctly smaller value than that of $\mathrm{PFM}_{2}$. It may be noted that physical variable 1 is very similar in definition to the generalized variance in multivariate statistics (Takeuchi \& Yanai, 1972) which is a measure of multivariate variability. Accordingly, the present conclusion implies that the generalized variance is a good measure of dispersion from the perceptual point of view as well.

(b) Judging from the fact that we need four correction classes in $\mathrm{QFM}_{1}$ solutions for both dot types, we infer that subjects utilize not only physical variable 1 but some physical properties of stimuli which cannot be made explicit at present.

(c) According to the present analysis PFM was found to be least descriptive of the data among the three types of representation models. Nonetheless, it is important to observe that QFM is far superior to UCM, since it means that PFM is capable of describing a large portion of the variance in the data.

\section{Discussion}

1. As is just mentioned, the psychophysical function model showed the worst fit among the three representation models. One probable reason for this may be that the present study obtained replications over different subjects rather than within a single subject. It is well recognized that the term "dispersion" is more equivocal than that of loudness or numerosity. Consequently, the possibility exists that relevant physical properties of stimuli in perception of dot dispersion might vary over subjects. This means that to find the true goodness of fit of PFM we need to analyze a set of data collected from an experiment where a single subject repeats trials many times. In such an experiment, however, it is difficult to obtain the statistical independence of observations. (For the problem of independence of each observation, see Takane and Carroll, 1981.)

2. The quasi-psychophysical function model we have proposed in this paper may sound like an arbitrary modification of the unconstrained model. From the point of view of MAICE, however, this is a natural restriction on UCM. First, no doubt there exist some physical properties in the dot pattern that elicit the subjective impression of dot dispersion. Therefore, at least some portion of the scale value estimated from UCM is to be accounted for in terms of those properties. This implies that $\mathcal{N}_{c}$ in QFM is apt to be smaller than $\mathcal{N}_{d}$ in UGM. That is, QFM hardly fails to give smaller AIC values than UCM. Second, correction parameters explicitly show us which stimuli deviate in what direction and how far from what PFM predicts. This may lead us to further investigations on reasons for those deviations.

However, there are at least two weaknesses in QFM. One is that grouping of stimuli into correction classes is done somewhat by trial and error, although the differences in value of the corresponding $d_{i}$ between UCM and PFM afford good information for classification and initial estimates of $C_{l}$. The other is that the 
model is not capable of predicting dispersion values of stimuli other than those actually used in the experiment while PFM can predict their values as well.

3. The present analysis was not able to clearly distinguish physical variable 1 and 2 in the degree of their importance in dispersion judgments. In order to compare the two physical variables in more detail it would be helpful to conduct the experiments several times using different sets of stimuli and different groups of subjects, and see if consistent results will be brought about. Or, if we can use a set of dot patterns which range over physical variable 1 , remaining approximately constant on physical variable 2 , then the correlation coefficient can be computed between obtained rank orders and the values of stimuli on physical variable 1. Similarly the correlation coefficient is obtained for physical variable 2. Then, these coefficients will help us determine which is the more relevant variable, although it may be difficult to collect such sets of stimuli.

4. We did not choose, as the representation model, Stevens' power law

$$
d_{i}=\alpha\left(s_{i k}+\gamma\right)^{4},
$$

where $\alpha$ and $A$ are constants. This does not mean that the power function is less suitable for the model of scaling dot dispersion. It is rather because in the framework of the present method Stevens' power function model is equivalent to Fechner's logarithmic function model if we employ the multiplicative error model (Takane, 1981)

$$
\left\{\begin{array}{l}
\lambda^{(l)}{ }_{i r}=d_{i r} e^{(l)}{ }_{i r} \\
\ln e^{(l)}{ }_{i r} \sim \mathcal{N}\left(0, \sigma^{2}\right)
\end{array}\right.
$$

instead of the additive error model defined by (9). This is shown by taking the $\log$ of (16) and substituting it into the $p^{(m)}{ }_{r}$ derived under the assumption of multiplicative error process (Takane \& Carroll, 1981).
5. We are currently attempting to scale similarities among schematic faces composed of polygons using much the same method and approach as taken in this study. In general, it is of interest to extend the present method to scaling such properties as randomness of scattered dots, stimulus complexity, symmetry, regurality, figural goodness, and so on. Clear definitions of these terms have not been given in objective measures of stimulus attributes or structures. Combination of our present method and Leeuwenberg's " coding theory" (Leeuwenberg, 1971, 1978) is promising in the study of psychophysical scaling of such properties.

\section{References}

Akaike, H. 1974 A new look at the statistical model identification. IEEE Transaclions on Automatic Control, 19, 716-723.

Indow, T., \& Ida, M. 1977 Scaling of dot numerosity. Perception \& Psychophysics, 22, 265276.

Leeuwenberg, E.L.J. 1971 A perceptual coding language for visual and auditory patterns. American Journal of Psychology, 84, 307-350.

Leeuwenberg, E.L.J. 1978 Quantification of certain visual pattern properties: Salience, transparency, and similarity. In E.L.J. Lecuwenberg \& H.F.J.M. Buffart (Eds.), Formal theories of visual perception. New York: Wiley. Pp. 277 298.

Luce, R. D., \& Galanter, E. 1963 Discrimination. In R. D. Luce, R. R. Bush \& E. Galanter (Eds.), Handbook of mathematical psychology. Vol. I. New York: Wiley. Pp. 191-243.

Takane, Y. 1981 Multidimensional successive categories scaling: A maximum likelihood method. Psychometrika, 46, 9-28.

Takane, Y., \& Carroll, J. D. 1981 Nonmetric maximum likelihood multidimensional scaling from directional rankings of similarities. $P s y$ chometrika, 46, 389-405.

Takane, Y., \& Sergent, J. 1983 Multidimensional scaling models for reaction times and same-different judgments. Psychometrika, 48, 393423.

Takeuchi, K., \& Yanai, H. 1972 The foundations of multivariate analysis: A unified approach by means of projection onlo linear subspace. Toyo-keizai- 
shimposha. (In Japanese)

Tone, K. 1981 BASIC. (Microcomputer programs series 1.) Baifu-kan. (In Japanese)
(Received Dec. 9, 1983; accepted

Nov. 10, 1984) 